\title{
Prevention of Health Hazards While Working with Chromium Compounds
}

\author{
${ }^{1}$ Angela A. Michailova, ${ }^{2}$ Elena E. Zinurova, ${ }^{3}$ Timofey Yu. Gumerov, ${ }^{4}$ Olga A. Reshetnik \\ 1,2 Kazan Federal University, Kremlyovskaya str., 18, 420008, Kazan, Russian Federation \\ ${ }^{3,4}$ Kazan National Research Technological University, K Marx str., 68, 420015, Kazan, Russian Federation \\ Email: lenazinurva@yandex.ru
}

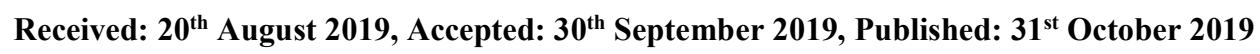

\begin{abstract}
This work presents a food product for the prevention of occupational diseases when working with chromium compounds. Efficiency consists in weakening the processes of allergization and sensitization of the human body when exposed to chromium compounds, as well as reducing occupational allergic diseases such as dermatitis, asthmatic bronchitis and bronchial asthma.

It was found that many types of industrial dust, aerosols and vapors have the ability to mechanically injure and cause irritation of the mucous membranes of the respiratory tract. Therefore, by the nature of the action on the respiratory system, they are divided into the following groups:

1. Substances with a pronounced sensitizing effect (rosin, some types of wood dust, bitumen).

2. Substances-sensitizers, which also have a local irritant effect, and some of them cause the development of pneumofibrosis and bronchial asthma (chromium, nickel chloride, chloramine, electric welding aerosol, formaldehyde, dust from flour, cotton, tobacco, wool and cement).

At the same time, depending on the chemical structure and physical properties of such allergens, damage to the respiratory apparatus in the form of chronic toxic or dust bronchitis, toxic pneumosclerosis or pneumoconiosis may initially be observed in the clinical picture. In the future, against the background of these diseases of the lungs, the development of bronchial asthma is possible.
\end{abstract}

\section{Keywords}

Chromium Intoxication, Occupational Diseases, Labor Protection, Cereal Product.

\section{Introduction}

In modern metallurgical plants, the dust factor is one of the leading ones. All stages of the technological process (crushing, mixing, sintering) are accompanied by significant dust emission of iron, chromium, manganese oxides (tens and hundreds of milligrams per $1 \mathrm{~m} 3$ of air). Chrome belongs to ferrous and base metals, in its pure form is one of the most durable metal elements. Industrial chromium poisoning is most often observed in leather, textile, printing, paper production, as well as in electrochromic metals.

Intoxication with chromium compounds is accompanied by suffocation, dysfunction of the respiratory system with widespread bronchospasm and increased secretion of the mucous membrane of the bronchioles, spasm.

Occupational bronchial asthma often occurs amid complete health during work, all of a sudden. Upon termination of contact with sensitizing substances passes. At first, the attack quickly stops when taking bronchodilators. Severe suffocation can provoke a previous rhinitis, hypothermia. In the clinical picture, two main periods can be distinguished: the onset and interictal.

For bronchial asthma, eosinophilia in the peripheral blood and the appearance of a small amount of vitreous sputum mucosa are very characteristic. More often they appear at the height of seizures, and may be absent in the interictal period. The nature of the course distinguishes between mild, moderate and severe degrees of bronchial asthma,

For the prevention of bronchial asthma, a thorough professional medical selection of people entering production where contact with industrial allergens is possible is of great importance. Therefore, the list of medical contraindications for working with substances with a sensitizing effect includes various diseases of both an allergic and non-allergic nature, which can contribute to the development of professional bronchial asthma.

Essential preventive measures for professional bronchial asthma are the early detection of the initial signs of the disease and rational employment outside contact with industrial allergens. In such cases, it is sometimes possible to prevent the further development of bronchial asthma and maintain the patient's ability to work.

\section{Materials and Methods}

In the modern automotive industry, the aviation industry, in the steel industry of the defense complex and the aerospace industry, chromium and steel based on it as an alloying agent are firmly in use. Chromium-based steels significantly increase the hardness of the finished product, alloys become more resistant to oxidation, which takes place at significantly higher temperatures, and are also mechanically more inert and resistant to friction. Chrome also found its use in the manufacture of particularly heat-resistant parts, such as gas turbine blades in the form of a compound of this metal with boron. The words "chrome" and "friction" are very related, and on its basis various grinding pastes are obtained, as well as solids for grinding and polishing various astronomical glasses and metal products [1,2].

Chromium compounds are widely used for the production of various paints and pigments (these are chromates of 
ammonium and alkali metals). Toxic compounds such as sodium and potassium dichromate are widely used as tannins in the leather industry. The compounds of this metal are treated with seeds; they are used as reagents in analytical chemistry and photographic work. From the above it becomes clear that the benefits and harms of chromium for humans are great, and if there is no need to talk about benefits, then it becomes necessary to consider its harmful effects [3].

Occupational diseases at work are caused by poisoning in the form of an aerosol of hexavalent chromium, which is constantly released from the molten surface of freshly cast metal and high-temperature slags. Occurrence of professional chromium intoxication is associated with labor activity at crushing and processing plants, as well as various open methods of mining ore containing this metal.

Harmful production factors are the main cause of poisoning. Of course, acute poisoning with soluble salts taken orally, is now very rare, as cases of clinical casuistry. The largest number of cases of poisoning by chromium and its compounds occurs due to its presence in the air of smelters and in the production of ore preparation. We are talking about contamination of the working area of grate platforms, on the control panel of thermal furnaces, in the crushing zone of the rock, on the dosage unit and in other places of steel and alloy production [4].

Exceeding the maximum permissible concentrations of this metal and compounds, as well as dust, is caused by various violations of the sealing of technological zones, casting of molten metal, loading of chromium alloys into cars and their transportation.

As with any other intoxication, the symptoms of chromium poisoning will depend on the route of ingestion or on the skin, on contact time, and on valency. The higher it is at the atom in the compound, the harder the signs of chromium poisoning. There is acute and chronic intoxication.

In acute poisoning, large doses of hexavalent chromium most often enter the body. If the drug is taken orally (for example, a solution of potassium dichromate), then one of the characteristic symptoms is severe pain in the epigastrium and abdomen, symptoms of gastrointestinal bleeding, which in severe cases is complicated by perforation of the intestine. After some time, sites of necrosis develop in the pancreas and liver, and the patient develops hemolysis of blood [5].

With prolonged contact with chromium compounds, of which chrompic dust has particularly pronounced toxic properties, chronic toxic bronchitis may occur with the subsequent development of pneumosclerosis and pulmonary emphysema. With sensitization to chromium, attacks of bronchial asthma occur.

When solutions get on the skin, they irritate, cauterize it, cause denaturation of skin proteins and mucous membranes. Prolonged contact with the skin leads to the formation of ulcers, for example, during prolonged contact with some types of concrete. When chromium compounds enter and are absorbed into the blood, hemolysis or massive disintegration of red blood cells occurs in the lumen of the vessels, as a result of hemolysis, a hemoglobin compound incapable of oxygen transfer or methemoglobin is formed.

Long-term exposure to compounds leads to the accumulation of this element mainly in the kidneys, liver tissues and endocrine glands. Since chromium and its compounds in the vast majority are excreted by the kidneys, their damage can be quite serious and lead to chronic renal failure [6].

\section{Research Results}

The harm to chromium for the body with prolonged local and aerogenic (inhalation) contact is the occurrence of cancerous tumors, and we are talking about an element that is hexavalent. Workers who came into contact with the pigments of this compound in production often came to the attention of surgeons in connection with various malignant tumors of the nose at the turn of the 19th and 20th centuries. Lung cancer is also much more likely to occur in the zone of unprotected production of chromium compounds, as well as when inhaling its dust.

Chromates cause irritation and cauterization of the mucous membranes and skin, which can lead to the development of ulcerative necrotic processes of the upper respiratory tract, mainly the nasopharynx, as well as ulceration of the skin. There are indications of a sensitizing effect of chromium and its compounds, which may lead to the appearance of allergic diseases - bronchial asthma and Quincke edema. During the pathological process, the following stages are distinguished $[7,8]$ :

- Nose pain, sore throat, mild cough; hyperemia of the nasal mucosa, serous discharge;

- Dry nose, nosebleeds, the formation of small crusts and ulcerations on the middle turbinates;

- Ulcerations of the mucous membrane become deeper and reach the cartilage, and finally, with the progression of the ulcerative process, the cartilage is gradually destroyed, which leads to perforation of the nasal septum.

Chromium poisoning is characterized by secondary changes in the bones and joints of the fingers, developing dermatitis, eczema and deep ulceration of the skin. In this case, skin lesions are slightly painful, therefore, often imperceptibly for the victim, they can lead to severe lesions of the underlying tissues. In such situations, the process extends to the interphalangeal joints, accompanied by significant destruction of the articular ends of the phalanges.

Primary care for chromium poisoning depends on the route of toxic compounds in the human body. So, when inhaling metal dust and damaging the nasal mucosa, protective washing of the nose with soapy water, lubrication of the nostrils with methyluracil and petrolatum ointment are necessary. With the development of asthma attacks, injectable use of aminophylline, atropine or platifillin is necessary.

Most often in everyday life there is a hit of chromium salt on the skin. In this case, a long rinse with warm water is required for at least 15 minutes, and then a bandage with ointment should be applied, which will prevent the cauterizing 
effect. If the solution gets into the eyes, a long rinse with water is also required for at least 15 minutes, followed by instillation of an albucide solution in the eyes to prevent a microbial secondary process.

It is very important to find out which chromium caused poisoning when taken orally. If this is a green compound of trivalent chromium, then you can not take on the removal of these salts even if it enters the gastrointestinal tract, since trivalent chromium is not toxic, and it is they that are part of picolinate. But such patients must be prescribed sorbents, activated carbon, since in addition to chromium, other harmful and toxic substances can also be present in the compounds.

If the poisoning occurred with potassium dichromate, then unitiol is administered intravenously as an antidote, in a dosage of $10 \mathrm{ml}$ of a $5 \%$ solution, every 8 hours, until the condition stabilizes. The average lethal dose for a person of soluble hexavalent chromium compounds is only 5 grams [9].

In production, technological measures give the best effect. These are equipment sealing, automation in production, compliance with forced ventilation standards, a separate workshop for working with chromium compounds, prerequisites for the neutralization of work clothes after shifts, washing, drying and dust removal. Where such measures cannot be applied due to technological reasons, it is necessary to use respirators, gloves, insulating gas masks. One of the main methods of medical prophylaxis is a periodic medical examination according to order No. 302n (as amended on February 6, 2018), since chromium is not only beneficial to humans, but also harmful: it is an allergen, a carcinogen, and an element whose compounds can cause severe poisoning up to lethal [10].

Hexavalent chromium, in comparison with trivalent, has more pronounced toxic and allergenic properties. Vapors of chromium and its compounds can enter the air of galvanic shops during chromium plating and the manufacture of catalysts containing chrompeak. In foundries, they appear in the production of high-quality steels; chrome is also part of some refractories. [eleven].

A healthy lifestyle, regular training and proper nutrition play an important role in reducing the risk and prevention of intoxication of compounds of chromium and other heavy metals [12-13].

According to the order No. 46n of the Ministry of Health and Social Development of the Russian Federation of February 16, 2009 (Appendix No. 2, registered with the Ministry of Justice on April 20, 2009, No. 13796), the Institute of Nutrition RAMS proposed a therapeutic and preventive diet No. 2a, for those who working under conditions of exposure to allergic substances (chromium and chromium-containing compounds). The diet is recommended under especially harmful working conditions to weaken the processes of allergization (sensitization) of the body. At the same time, hot first courses should be cereal, vegetable or milk, cooked on weak meat and fish broths. The second dishes should be cooked in boiled, steam form, as well as baked and stewed without prior frying. Fried foods are not allowed [14].

The authors of the work proposed a cereal product for food working with chromium-containing compounds, for which a RF patent No. 2681104 was obtained (published on March 4, 2019, bull. No. 7 g). Table 1 shows the ingredient composition and energy values of this product.

\begin{tabular}{|l|l|l|l|l|l|}
\hline \multirow{2}{*}{ Ingredients } & \multirow{2}{*}{ Weight, $\mathrm{g}$} & \multicolumn{3}{|c|}{ Content, $\mathrm{i}$} & \multirow{2}{*}{ Energy value, kcal } \\
\cline { 3 - 5 } & & Proteins & \multicolumn{1}{|c|}{ Fats } & Carbohydrates & \\
\hline oat bran & 135 & 17,82 & 6,21 & 64,9 & 408,29 \\
\hline fine fiber & 60 & 9,6 & 14,1 & 2,28 & 111,8 \\
\hline flax flour & 50 & 12,5 & 2,5 & 6,5 & 152,5 \\
\hline whole lentil flour & 85 & 17,8 & 44,9 & 8,9 & 250,8 \\
\hline freshly chopped ginger & 35 & 0,63 & 0,28 & 6,3 & 28 \\
\hline swede & 95 & 1,14 & 0,1 & 7,32 & 35,2 \\
\hline anise seeds & 30 & 5,3 & 4,8 & 15 & 101,1 \\
\hline dried pears & 65 & 1,4 & 0,4 & 40,7 & 175,5 \\
\hline prunes & 70 & 1,6 & 0,5 & 40,3 & 179,2 \\
\hline chopped turnip & 70 & 1,1 & 0,1 & 4,3 & 22,4 \\
\hline fresh rose hips & 50 & 0,8 & 0,4 & 11,2 & 54,5 \\
\hline dried seaweed & 50 & 28,7 & 3,9 & 12 & 145 \\
\hline Rowan & 15 & 0,2 & 0 & 1,3 & 7,5 \\
\hline fresh dill & 30 & 0,8 & 0,2 & 1,9 & 12 \\
\hline green pea & 60 & 3 & 0,1 & 5 & 33 \\
\hline linseed oil & 70 & 0 & 69,9 & 0 & 628,6 \\
\hline spinach & 30 & 0,9 & 0,1 & 0,6 & 6,9 \\
\hline TOTAL & 1000 & 103,29 & 148,49 & 228,5 & 2352,29 \\
\hline Total & 100 & 10,33 & 14,85 & 22,85 & 235,23 \\
\hline Total & 30 & 3,09 & 4,45 & 6,86 & 70,57 \\
\hline
\end{tabular}

Table 1: The Composition of the Cereal Product for the Prevention of Occupational Diseases when Working with Chromium Compounds

The proposed food product is enriched with alkaline ingredients with a high content of lecithin, proteins with a large 
amount of sulfur-containing amino acids, vitamins C, P, PP, E, A, trace elements Ca, Mg, S, as well as pectin and organic acids, which meets the requirements of the above order Ministry of Health and Social Development of the Russian Federation.

The selected optimal composition of the ingredients will help to weaken the body's allergization (sensitization) processes by inhibiting the oxidation and decarboxylation of tryptophan to serotonin, histidine to histamine, tyrosine to tyramine and to increase the methylation of biogenic amines to an inactive state.

The process of preparing a cereal product consists in preparing a mixture of dry and liquid components with the addition of linseed oil. The resulting mass is kneaded to the most uniform state, distributed in a form, kept for 15-20 minutes and baked in an oven at a temperature of 180 degrees for 13-15 minutes. After that, the obtained cereal cake is cooled and cut into bars weighing 30 grams.

\section{Conclusions}

Thus, the composition of this product includes ingredients that will help reduce occupational allergic diseases, such as dermatitis, bronchial asthma and asthmatic bronchitis, effectively adsorb and remove them from the body of chromium-containing compounds. The proposed food product does not contain immunogenic xenobiotics, can be an additional source of necessary food components and provide a lack of energy value in the nutrition of individuals when working with chromium-containing compounds.

\section{Acknowledgements}

The work is performed according to the Russian Government Program of Competitive Growth of Kazan Federal University.

\section{References}

1. Bindyuk T.Ya. Occupational diseases. Textbook for students of higher educational institutions studying in the specialty 033300 "Life Safety" / T.Ya. Bindyuk, O.V. Countless. Publishing house "Nikolaev", Balashov, Saratov region. - 2007. - $128 \mathrm{p}$.

2. Erofeeva T.I. Chemical elements in the modern world: a teaching aid. - 2009. -- 362 p.

3. Kudryavtseva V.T. Chemistry: teaching aid. - 2009. - 72 p.

4. Kunin A.M. The effect of hexavalent and trivalent chromium on representative hydrobionts of the model food chain. Abstracts at the conference Problems of hydroecology at the turn of the century. - 2000. - P. 228-229.

5. Levina E.N. General toxicology of metals. - 1972. - P.82-87.

6. Popovkin N.L. The effect of chromium on the functional ability of the kidneys in people employed in chromium production and residents of the technogenic zone. All-Union Congress of Laboratory Physicians. - 1991. - $71 \mathrm{p}$.

7. Hayes R.B. Aspects Chromium // Biol a Environ.- 1982.- P. 221-247.

8. Borska L., Fiala Z., Smejkalova J., Tejral J. Health risk of occupational exposure, in welding processes // Acta Medica. 2003. Vol. 46, No 1. P. 25-29.

9. GN 2.2.5.1313-03. Maximum allowable concentration (MPC) of harmful substances in the air of the working area. - 2003. -- $185 \mathrm{p}$.

10. Izmerov N.F. National project "Health" the role of occupational medicine. // Honey. tr and prom. eco-friendly. 2007. - No. 12. - P. 4-8.

11. Mamyrbaev A.A., Zasorin B.V., Malyshkina C.B. The combined effect of chemical and physical factors on the health of workers working in chrome production. // Honey. tr and prom. eco-friendly. - 2006. - No. 14. P. 17-19.

12. Muraveva E.V., Stepuschenco O.A., Sibgatulina D. Sh. Provision of ecological safety of water supplying system of industrial enterprises. Proceedings of the Six International Environmental Congress (Eighth International Scientific - Technical Conference) "Ecology and Life Protection of Industrial-Transport Complex". - 2017. -- P.401. 13. Smolentsev S. Yu., Volkov A.Kh., Papunidi E. K., Yusupova G. R., Nikolaev N.V., Larina Yu. V, Romanova N. K. Meat Productivity of Cattle Depending on the Composition of the Ration. Research Journal of Pharmaceutical, Biological and Chemical Sciences. - 2018. No. 9. - P.1247-1251.

14. Mingaleeva Z.Sh., Agzamova L.I., Starovoitova O.V. Determination of the optimal concentration of antioxidant additives in the production of deep-fried pastry. // Natural and technical sciences. - 2015. - No. 5. - P. $171-177$. 